\title{
Numerical Calculation of the Essential Spectrum of a Laplacian
}

\author{
J. W. Neuberger and R. J. Renka
}

\section{CONTENTS}

1. Introduction

2. Background on General Laplacians

3. Rooms and Passages Region

4. Results

5. Numerical Techniques

6. Conclusion

References
We consider a bounded Rooms and Passages region $\Omega$ on which the negative Neumann laplacian (restricted to the orthogonal complement of the constant functions) does not have a compact inverse and hence has an essential spectrum. We try to understand how such spectra may be approximated by results from a sequence of finite-dimensional problems. Approximations to this laplacian on finite-dimensional structures have only eigenvalues for spectra. Our strategy is to attempt to discern how results on increasingly better approximating structures point to spectral results in the limiting case.

\section{INTRODUCTION}

The determination and understanding of spectra of linear differential operators is important in quantum mechanics as well as in several areas of mathematics. When an operator on a Hilbert space has a compact self-adjoint inverse, it is well-known that a complete set of eigenfunctions exists for which the corresponding eigenvalues form a simple unbounded infinite sequence. In case such an operator has a bounded self-adjoint inverse which is not compact, it also has an essential spectrum. For example, the set of eigenvalues may have a nonzero limit point. Now to obtain numerical approximations to such spectra one must make finite-dimensional approximations to both an operator and to a region on which it acts. Such a finite-dimensional approximation leads only to eigenvalues since essential spectra are impossible on a finite-dimensional space. How might eigenvalue patterns for finite-dimesional approximations point to continuous spectra? In this note we make a case study of such a phenomenon. For this case study we choose a spectral problem for the negative Neumann laplacian on a 'Rooms and Passages' region $\Omega$ in $R^{2}$. For this bounded region $\Omega$ the Sobolev space $H^{1,2}(\Omega)$ is not compactly embedded in $L_{2}(\Omega)$ 
and we will see that the corresponding self-adjoint inverse negative Neumann laplacian is not compact.

Suppose $T$ is a self-adjoint linear transformation on a Hilbert space $H$. The spectrum of $T$ consists of all numbers $\lambda$ so that either $T-\lambda I$ does not have an inverse or else it does but $(T-\lambda I)^{-1}$ is not a member of $L(H, H)$. From [Edmunds and Evans 1987, Chapter IX, Theorems 1.3-1.6], one has a division of the spectrum into two classes: The first set consists of all eigenvalues of $T$ with finite multiplicity; the second set consists of all numbers $\lambda$ for which there is a singular sequence, i.e., a sequence $\left\{x_{j}\right\}_{j=1}^{\infty}$ of unit vectors in $H$ which has no convergent subsequence and for which

$$
\left\|(T-\lambda I) x_{j}\right\|_{H} \rightarrow 0 \quad \text { as } j \rightarrow \infty .
$$

This second set is called the essential spectrum of $T$.

In [Hempel et al. 1991] it is shown that, given a closed subset $S$ of $[0, \infty)$, one may choose a Rooms and Passages or Comb region so that the essential spectrum of the corresponding negative Neumann laplacian is precisely $S$. In the present work we deal with a single example which is of interest in the theory of Sobolev embeddings. From the results in [Hempel et al. 1991] we are assured that there is a great variety of possibilities for essential spectra. Our code, or one similar, might be used to investigate some of these. In particular it would be interesting to understand how the spectra of finitedimensional approximations behave when the essential spectrum being approximated is an interval.

\section{BACKGROUND ON GENERAL LAPLACIANS}

Suppose $H$ is a Hilbert space with norm $\|\cdot\|_{H}, H^{\prime}$ is a dense linear subspace of $H$ and $\|\cdot\|_{H^{\prime}}$ is a second norm defined on $H^{\prime}$ such that

$$
\|x\|_{H} \leq\|x\|_{H^{\prime}} \quad \text { for } x \in H^{\prime}
$$

and that $H^{\prime}$ is also a Hilbert space with this second norm. There are two linear transformations of interest in this setting. The first is $I_{H^{\prime}, H}$, the identity transformation considered as a transformation from $H^{\prime}$ to $H$. The second transformation is called $M$ and is described as follows; see [Beurling 1989, page 209] or [Neuberger 1997, Chapter 5], for example.
For $y \in H$ define $f \in H^{*}$ so that

$$
f(x)=\langle x, y\rangle_{H} \quad \text { for } x \in H .
$$

Denote by $g$ the restriction of $f$ to $H^{\prime}$. Then

$$
|g(x)|=\left|\langle x, y\rangle_{H}\right| \leq\|x\|_{H}\|y\|_{H} \leq\|x\|_{H^{\prime}}\|y\|_{H}
$$

for $x \in H^{\prime}$. Hence there is a unique element $z \in H^{\prime}$ such that

$$
g(x)=\langle x, z\rangle_{H^{\prime}} \quad \text { for } x \in H^{\prime} .
$$

Define

$$
M y=z .
$$

From either of the above two references or by an easy observation one has the following properties of $M$ :

(i) $\langle M x, y\rangle_{H}=\langle x, M y\rangle_{H}, x, y \in H$.

(ii) $\langle M x, y\rangle_{H^{\prime}}=\langle x, M y\rangle_{H^{\prime}}, x, y \in H^{\prime}$.

(iii) $|M|_{L\left(H, H^{\prime}\right)} \leq 1$.

(iv) $R(M)$, the range of $M$, is dense in $H$.

(v) $M^{-1}$ exists.

One calls $M^{-1}$ the laplacian associated with the pair $\left(H, H^{\prime}\right)$.

Theorem. $I_{H^{\prime}, H}$ is compact if and only if $M$ is compact as a member of $L(H, H)$.

Proof. Suppose $I_{H^{\prime}, H}$ is compact. Denote by $\left\{x_{k}\right\}_{k=0}^{\infty}$ a bounded sequence in $H$. Then $\left\{M x_{k}\right\}_{k=0}^{\infty}$ is a bounded sequence in $H^{\prime}$ and hence it has a convergent subsequence in $H$. Therefore $M$ is compact.

Now suppose that $M$ is compact as a member of $L(H, H)$. Denote by

$$
\left\{x_{k}\right\}_{k=0}^{\infty}
$$

a bounded sequence in $H^{\prime}$. We want to show that this sequence has a convergent subsequence in $H$. To this end note that $(2-1)$ is also bounded in $H$ and hence has a subsequence (also denoted by $(2-1)$ ) which converges weakly to an element $x \in H$. Denote by $\alpha$ a number so that

$$
\left\|x_{k}\right\|_{H^{\prime}} \leq \alpha, \quad \text { for } k=1,2, \ldots
$$

Denote by $\left\{\varphi_{j}\right\}_{j=0}^{\infty}$ an orthonormal basis of $H$ consisting of eigenfunctions of $M$ so that its corresponding sequence $\left\{\lambda_{j}\right\}_{j=1}^{\infty}$ of eigenvalues is a nonincreasing sequence converging to 0 . 
Note that if $N$ is a positive integer then

$$
\begin{aligned}
\left\|x-x_{k}\right\|_{H}^{2}= & \sum_{j=1}^{\infty}\left\langle x-x_{k}, \varphi_{j}\right\rangle_{H}^{2} \\
\leq & \sum_{j=1}^{N}\left\langle x-x_{k}, \varphi_{j}\right\rangle_{H}^{2}+2 \sum_{j=N+1}^{\infty}\left\langle x, \varphi_{j}\right\rangle_{H}^{2} \\
& +2 \sum_{j=N+1}^{\infty}\left\langle x_{k}, \varphi_{j}\right\rangle_{H}^{2} .
\end{aligned}
$$

Now if each of $j$ and $k$ is a positive integer,

$$
\left\langle x_{k}, \varphi_{j}\right\rangle_{H}=\left\langle x_{k}, M \varphi_{j}\right\rangle_{H^{\prime}}=\lambda_{j}\left\langle x_{k}, \varphi_{j}\right\rangle_{H^{\prime}}
$$

so that

$$
\begin{aligned}
\sum_{j=N+1}^{\infty}\left\langle x_{k}, \varphi_{j}\right\rangle_{H}^{2} & =\sum_{j=N+1}^{\infty} \lambda_{j}^{2}\left\langle x_{k}, \varphi_{j}\right\rangle_{H^{\prime}}^{2} \\
& =\sum_{j=N+1}^{\infty} \lambda_{j}\left\langle x_{k}, \beta_{j}\right\rangle_{H^{\prime}}^{2}
\end{aligned}
$$

where

$$
\beta_{j}=\varphi_{j} /\left\|\varphi_{j}\right\|_{H^{\prime}}=\sqrt{\lambda_{j}} \varphi_{j} \quad \text { for } j=1,2, \ldots
$$

since

$$
\left\|\varphi_{j}\right\|_{H^{\prime}}^{2}=1 / \lambda_{j} \quad \text { for } j=1,2, \ldots
$$

Then

$$
\begin{gathered}
\sum_{j=N+1}^{\infty} \lambda_{j}\left\langle x_{k}, \beta_{j}\right\rangle_{H^{\prime}}^{2} \leq \lambda_{N+1} \sum_{j=N+1}^{\infty}\left\langle x_{k}, \beta_{j}\right\rangle_{H^{\prime}}^{2} \\
\leq \lambda_{N+1} \sum_{j=1}^{\infty}\left\langle x_{k}, \beta_{j}\right\rangle_{H^{\prime}}^{2}=\lambda_{N+1}\left\|x_{k}\right\|_{H^{\prime}}^{2} \leq \lambda_{N+1} \alpha^{2} .
\end{gathered}
$$

Now suppose that $\varepsilon>0$. Pick $N$ such that

$$
2 \lambda_{N+1} \alpha^{2}<\varepsilon / 3
$$

and

$$
2 \sum_{j=N+1}^{\infty}\left\langle x, \varphi_{j}\right\rangle_{H}^{2}<\varepsilon / 3 .
$$

Pick $N^{\prime}$ so that if $k>N^{\prime}$ then

$$
\left\langle x-x_{k}, \varphi_{j}\right\rangle_{H}^{2} \leq \varepsilon /(3 N) \text { for } j=1,2, \ldots, N .
$$

Then

$$
\left\|x-x_{k}\right\|_{H}^{2}<\varepsilon \text { for } k>N^{\prime} .
$$

Hence $\left\{x_{k}\right\}_{k=1}^{\infty}$ converges in $H$ to $x$.

\section{ROOMS AND PASSAGES REGION}

For $\Omega$ a bounded region in $R^{2}$, take $H=L_{2}(\Omega)$ and $H^{\prime}$ to be the Sobolev space $H^{1,2}(\Omega)$ (see [Adams 1975; Edmunds and Evans 1987] for background on Sobolev spaces). Denote by $M$ the transformation associated with the pair $\left(H, H^{\prime}\right)$ as above. We are interested in a numerical indication of the spectrum of $M$ in a case where $\Omega$ is chosen so that $M$ is not compact. We actually present approximations to the spectrum of $M^{-1}-I$ (which corresponds to the classical negative Neumann laplacian).

Our region of interest is a Rooms and Passages domain taken from [Edmunds and Evans 1987, p. 272]. It is a connected bounded region consisting of a union of infinitely many squares $\left\{R_{k}\right\}_{k=1}^{\infty}$ (rooms) and infinitely many rectangles $\left\{P_{k}\right\}_{k=1}^{\infty}$ (passages) arranged in an alternating series. The heights of $R_{1}, P_{1}, R_{2}, P_{2}, R_{3}, \ldots$ are $1, \alpha, \alpha^{2}, \alpha^{3}, \alpha^{4}, \ldots$, the widths of $R_{1}, R_{2}, R_{3}, \ldots$ are $1, \alpha^{2}, \alpha^{4}, \ldots$, and the widths of $P_{1}, P_{2}, P_{3}, \ldots$ are $\alpha^{3}, \alpha^{9}, \alpha^{15}, \ldots$, where $\alpha$ is chosen to be $2^{-1 / 6}$. All the sides are parallel to the axes and all the rectangles are centered about a horizontal line. Theorem 4.21 in [Edmunds and Evans 1987, page 273] asserts that for this region $\Omega$, $H^{1,2}(\Omega)$ is not compactly embedded in $L_{2}(\Omega)$, i.e., that $I_{H^{\prime}, H}$ is not compact and hence neither is $M$.

In our discretization of $\Omega$, we refer to

$$
\Omega_{1}=R_{1} \cup P_{1} \cup R_{2}
$$

as level 1 and

$$
\Omega_{n}=\Omega_{n-1} \cup P_{n} \cup R_{n+1}
$$

as level $n$ for $n \geq 2$.

\section{RESULTS}

Figure 1 displays computed values of the smallest 12 eigenvalues of the negative Neumann laplacian on each of the first seven levels $\Omega_{1}, \ldots, \Omega_{7}$. The values are depicted as graphs of the $k$-th smallest eigenvalue versus level number $n$ for $k=1, \ldots, 12$ and $n=1, \ldots, 7$. Similarly, Figure 2 displays the smallest six computed eigenvalues on each of the first eight levels.

For $n$ and $k$ positive integers, denote eigenvalue $k$ on level $n$ by $\lambda_{k, n}$ for the negative Neumann laplacian on $\Omega_{n}$. We offer the following hypothesis based 


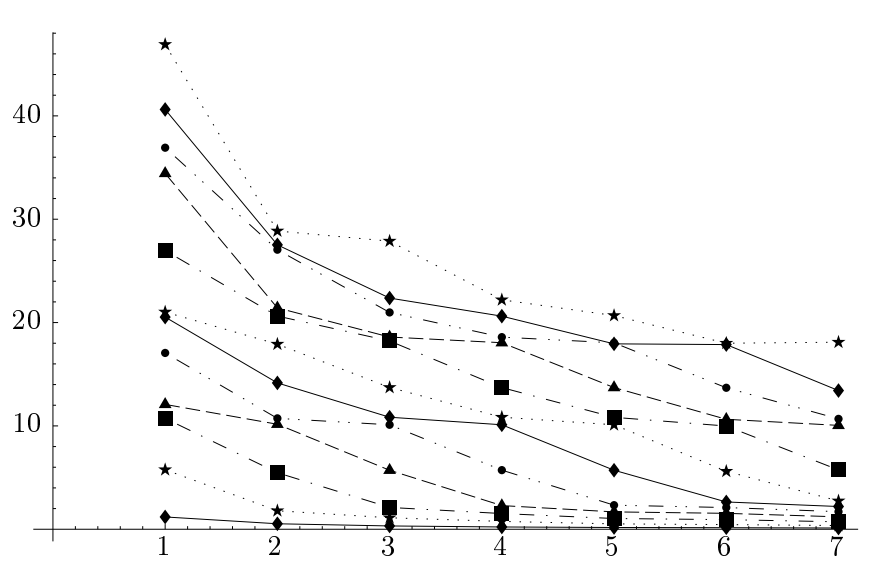

FIGURE 1. Lowest 12 eigenvalues plotted versus level.

on these graphs and our examination of contour plots of corresponding eigenfunctions.

\section{Hypothesis 1.}

1. $\lambda^{(m)}=\lim _{k \rightarrow \infty} \lambda_{k+m, k}$ exists for $m=1,2, \ldots$, and $\left\{\lambda^{(m)}\right\}_{m=1}^{\infty}$ is nondecreasing and unbounded.

2. $\lambda_{k}=\lim _{n \rightarrow \infty} \lambda_{k, n}$ exists and $\left\{\lambda_{k}\right\}$ is increasing.

3. $\lambda_{0}=\lim _{k \rightarrow \infty} \lambda_{k}$ exists.

4. $\lambda_{1}<\lambda_{2}<\cdots<\lambda_{0} \leq \lambda^{(1)} \leq \lambda^{(2)} \leq \cdots$.

The point $\lambda_{0}$ appears to be a point of essential spectrum. Figures $3-8$ depict contour plots of the computed eigenfunctions associated with $\lambda_{k+1, k} \approx 5.7$ for $k=1, \ldots, 6$. These clearly indicate convergence as $k \rightarrow \infty$. Plots of the eigenfunctions associated with $\lambda_{k+2, k} \approx 10.1$ (not included here) also indicate convergence, and we assume that this pattern applies to $\lambda_{k+m, k}$ for all $m$. Less compelling, but probably true, is the proposition that the eigenfunctions associated with $\lambda_{k, n}$ converge to distinct limits as $n \rightarrow \infty$ for $k=1,2, \ldots$. Our evidence for the case

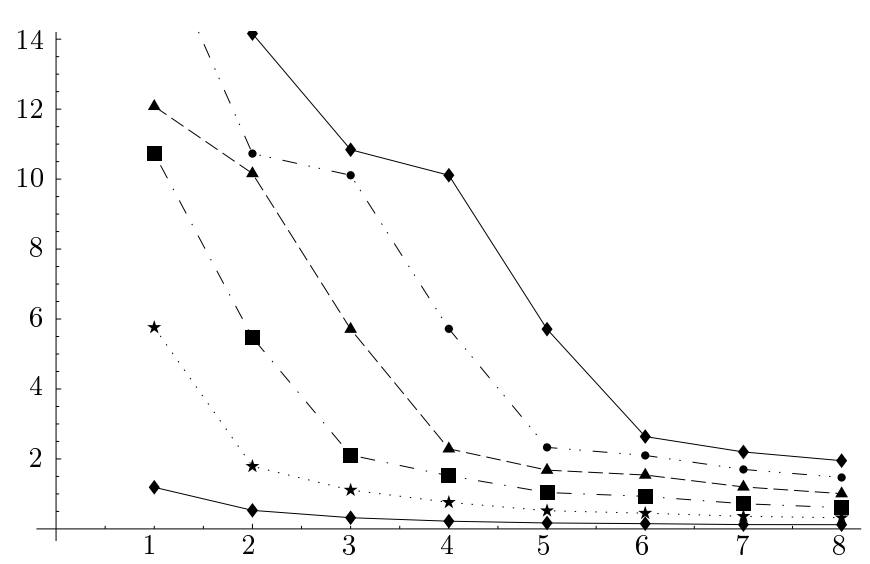

FIGURE 2. Lowest 6 eigenvalues plotted versus level.

$k=2$ is depicted in Figure 9, which we explain now. Contour plots for the second eigenfunction at various levels are similar to those in Figures $3-8$ in that they exhibit symmetry about the centerline, but they differ greatly in that they show considerable variation in the smaller passages at higher levels. This variation is illustrated in Figure 9 which contains centerline plots for eigenfunction 2 at levels 1 through 7 , the leftmost graph being for level 1, the next for level 2 and so on. The horizontal axis corresponds to distance along the centerline of the region. It seems like these plots point to a singularity at the right end of the full Room and Passage region. It is not clear to us whether $\lambda_{2}$ is an eigenvalue or a point of essential spectrum. If the sequence of eigenfunctions for the second eigenvalue converge, as the level increases, to an $L_{2}$ function, then this $L_{2}$ function is likely to be an eigenfunction for eigenvalue $\lambda_{2}$; if this sequence of eigenfunctions converges, in some sense, to a function which is not $L_{2}$ or else does not

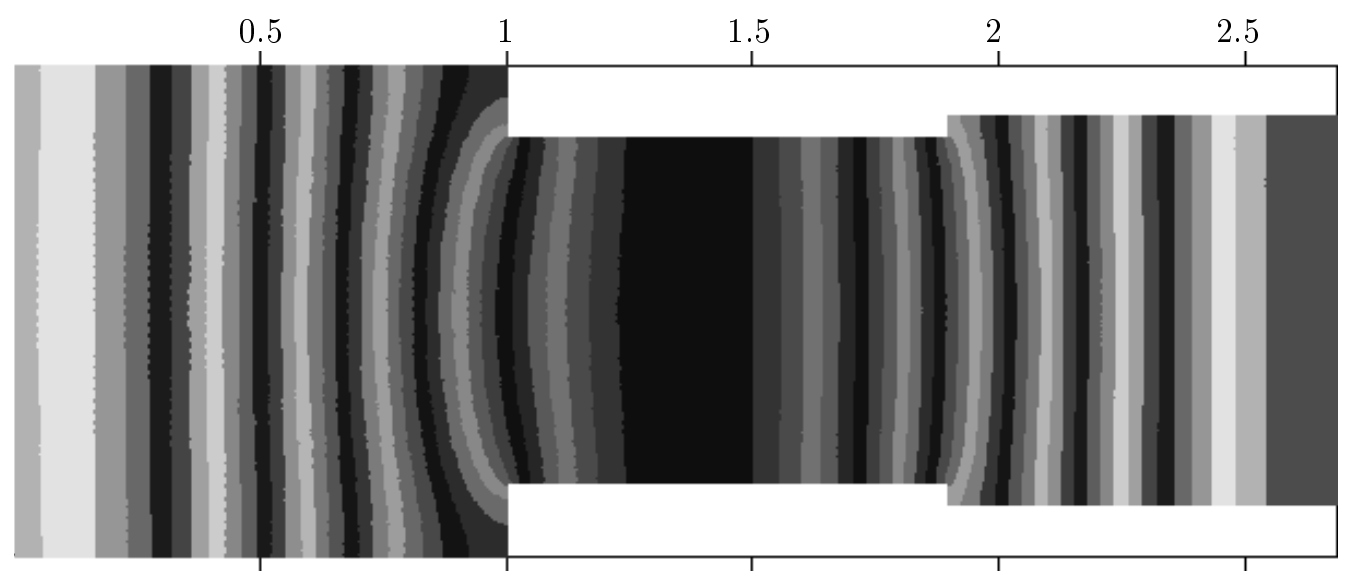

FIGURE 3. Eigenfunction 2, level 1. 


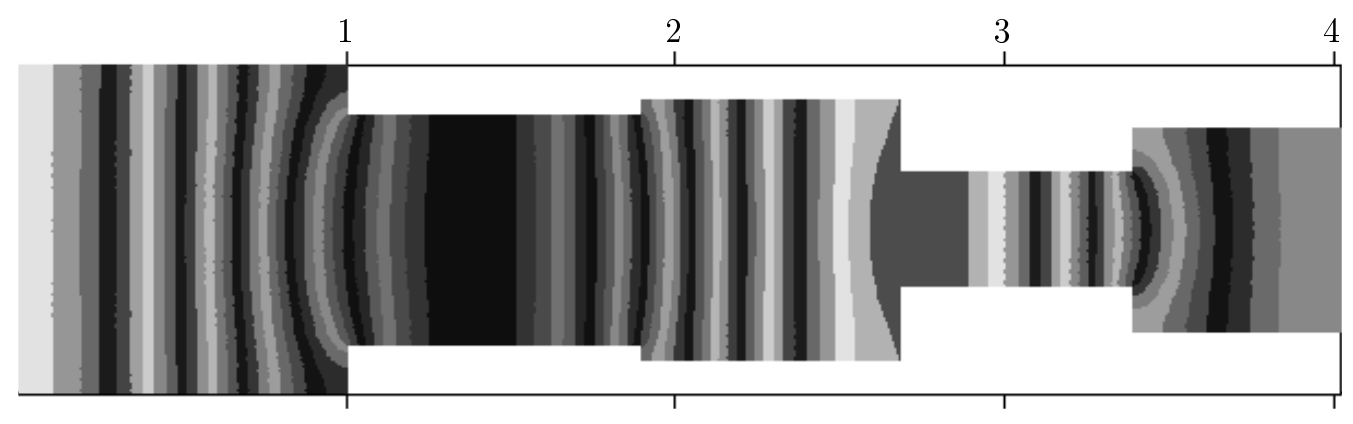

FIGURE 4. Eigenfunction 3, level 2.

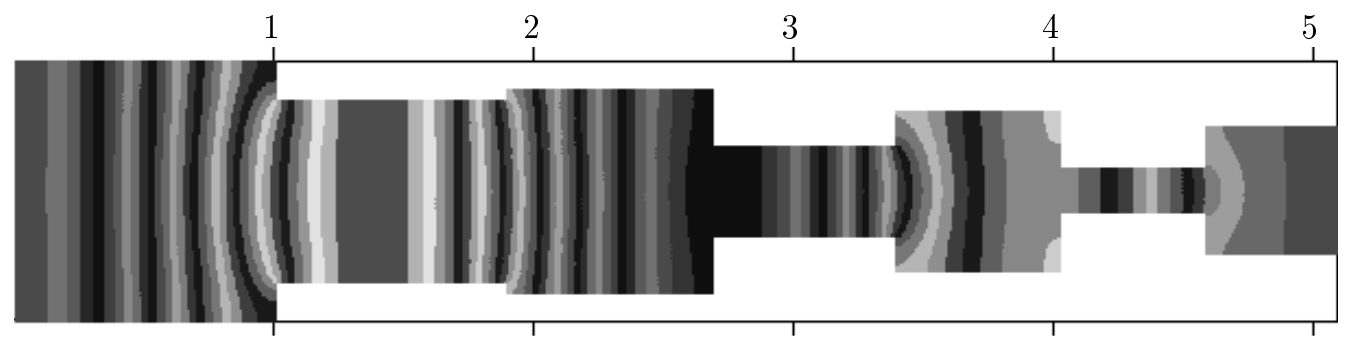

FIGURE 5. Eigenfunction 4 , level 3.

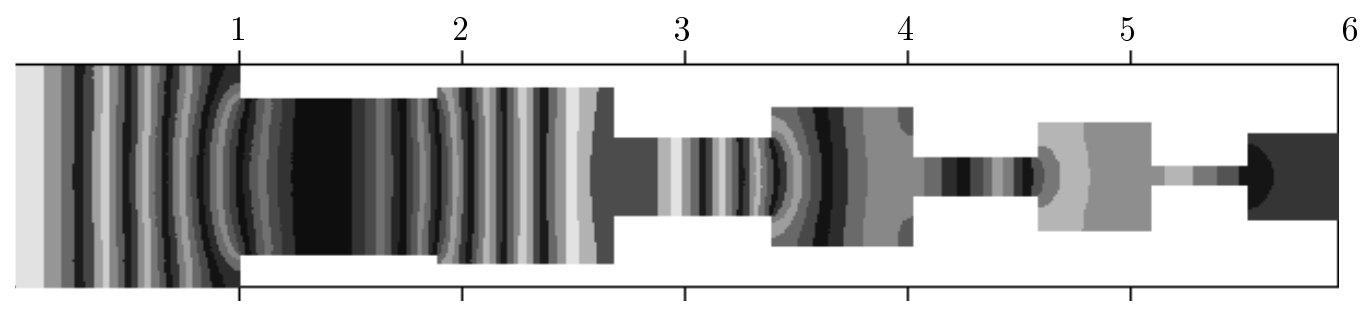

FIGURE 6. Eigenfunction 5, level 4.

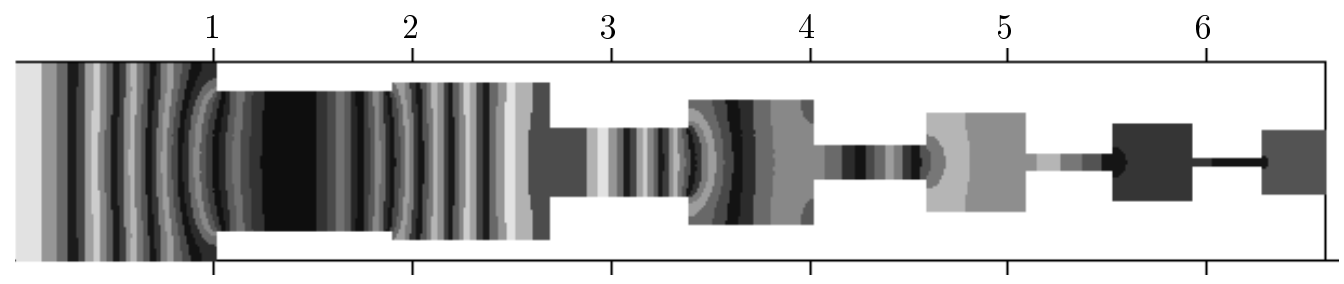

FIGURE 7. Eigenfunction 6, level 5.

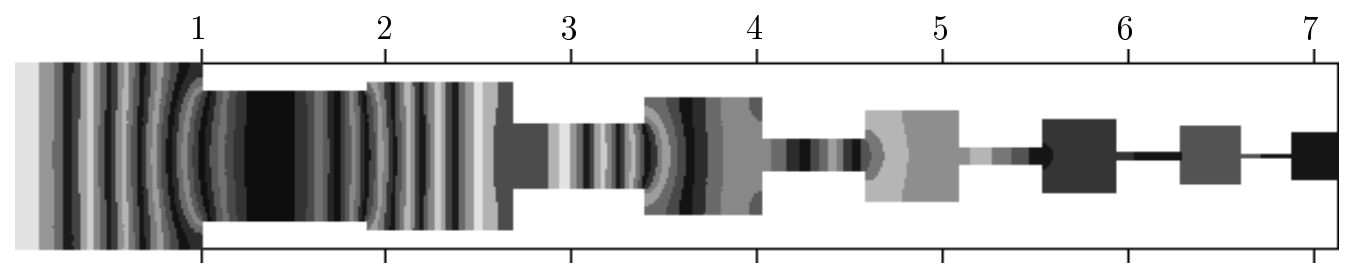

FIGURE 8. Eigenfunction 7, level 6 . 


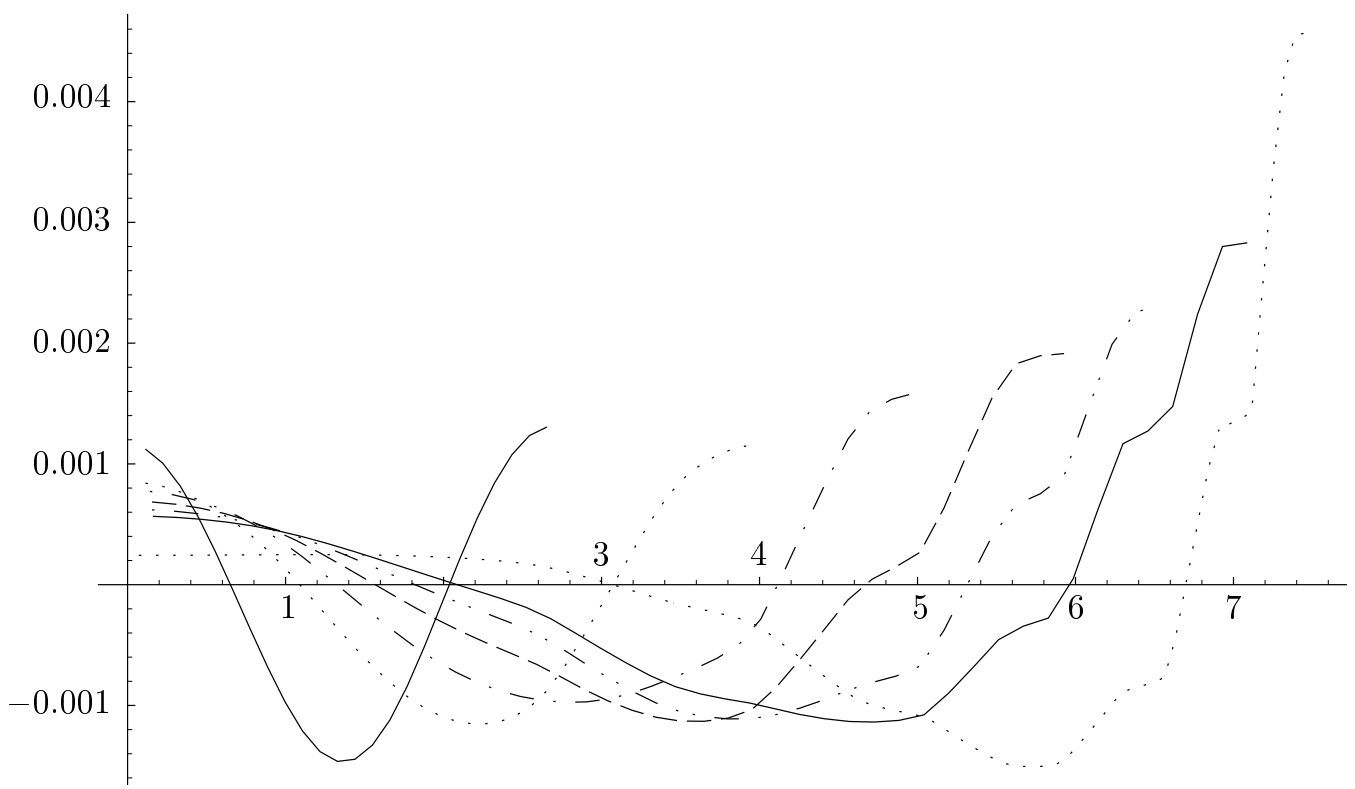

FIGURE 9. Eigenfunction 2, Levels 1-7.

converge in any reasonable sense, then $\lambda_{2}$ is almost certainly a point of essential spectrum. The same considerations apply to $\lambda_{k}, k=1,2, \ldots$, but we have no particular reason to believe that the situation is similar for all these cases.

We offer the following alternative:

\section{Hypothesis 2.}

1. $\lambda^{(m)}=\lim _{k \rightarrow \infty} \lambda_{k+m, k}$ exists for $m=1,2, \ldots$, and $\left\{\lambda^{(m)}\right\}_{m=1}^{\infty}$ is nondecreasing and unbounded. 2. $\lambda_{k}=\lim _{n \rightarrow \infty} \lambda_{k, n}=0$ for all $k$.

According to [Hempel et al. 1991], for some regions, 0 is the only point of essential spectrum. Evidence from Figure 2 does not, in our judgement, completely preclude this possibility in the present case.

\section{NUMERICAL TECHNIQUES}

Our method of finding eigenvalues and eigenvectors has its roots in the earlier works [Neuberger and Noid 1987; Lapidus, Neuberger, Renka, and Griffith 1996]. Our computed results are based on an inverse power method with deflation and a multigrid/SOR linear system solver. Our code constructs a uniform rectangular finite difference grid on any region which can be represented as a union of rectangles with sides parallel to the axes. The standard fivepoint difference operator is used to approximate the negative laplacian. The code allows for zero Dirichlet boundary conditions on an arbitrary portion of the boundary. We used this capability to more efficiently compute the odd eigenfunctions (those with nodal lines on the centerline of $\Omega$ ) by approximating just half of the domain.

The inverse power method is described as follows. Let $T$ be a symmetric positive definite linear transformation on $\mathbb{R}^{N}$ with eigenvalues

$$
0<\lambda_{1} \leq \lambda_{2} \leq \cdots \leq \lambda_{N},
$$

and denote the corresponding sequence of orthonormal eigenvectors by $\left\{\boldsymbol{u}_{i}\right\}_{i=1}^{N}$ so that $\boldsymbol{T} \boldsymbol{u}_{i}=\lambda_{i} \boldsymbol{u}_{i}$ and $\boldsymbol{u}_{i}^{T} \boldsymbol{u}_{j}=\delta_{i j}$ for $1 \leq i, j \leq N$. Then $\boldsymbol{T}^{-1}$ has eigenvalues $\left\{\lambda_{i}^{-1}\right\}_{i=1}^{N}$ with the same eigenvectors. Since the eigenvectors span $\mathbb{R}^{N}$, an arbitrary vector $\boldsymbol{x}_{0}$ may be written $\boldsymbol{x}_{0}=\sum_{i=1}^{N} \alpha_{i} \boldsymbol{u}_{i}$, and $m$ applications of $\boldsymbol{T}^{-1}$ to $\boldsymbol{x}_{0}$ results in

$$
\begin{aligned}
\boldsymbol{T}^{-m} \boldsymbol{x}_{0} & =\sum_{i=1}^{N} \alpha_{i} \boldsymbol{T}^{-m} \boldsymbol{u}_{i}=\sum_{i=1}^{N} \alpha_{i} \lambda_{i}^{-m} \boldsymbol{u}_{i} \\
& =\lambda_{1}^{-m}\left(\alpha_{1} \boldsymbol{u}_{1}+\sum_{i=2}^{N} \alpha_{i}\left(\lambda_{1} / \lambda_{i}\right)^{m} \boldsymbol{u}_{i}\right) .
\end{aligned}
$$

If $\alpha_{1} \neq 0$ and $\lambda_{1}<\lambda_{2}$, the above expression (suitably scaled) converges to a multiple of the dominant eigenvector $\boldsymbol{u}_{1}$ as $m \rightarrow \infty$.

The power method applied to $T^{-1}$ consists of solving the sequence of linear systems

$$
\boldsymbol{T} \boldsymbol{x}_{m+1}=\boldsymbol{x}_{m} \quad \text { for } m=0,1, \ldots,
$$

resulting in $\boldsymbol{x}_{m}=\boldsymbol{T}^{-m} \boldsymbol{x}_{0}$. Approximations to the dominant eigenvalue $\lambda_{1}^{-1}$ are obtained by computing 
the Rayleigh quotients $r_{m}=\left(\boldsymbol{x}_{m}^{T} \boldsymbol{T}^{-1} \boldsymbol{x}_{m}\right) /\left(\boldsymbol{x}_{m}^{T} \boldsymbol{x}_{m}\right)$ $=\boldsymbol{x}_{m}^{T} \boldsymbol{x}_{m+1} / \boldsymbol{x}_{m}^{T} \boldsymbol{x}_{m}$. Using orthogonality of the eigenvectors, we have

$\boldsymbol{x}_{m}^{T} \boldsymbol{x}_{m+1}=\sum_{i=1}^{n} \alpha_{i}^{2} \lambda_{i}^{-2 m-1}$ and $\boldsymbol{x}_{m}^{T} \boldsymbol{x}_{m}=\sum_{i=1}^{n} \alpha_{i}^{2} \lambda_{i}^{-2 m}$,

from which it follows that

$$
r_{m}=\lambda_{1}^{-1}\left(\frac{\alpha_{1}^{2}+\sum_{i=2}^{n} \alpha_{i}^{2}\left(\lambda_{1} / \lambda_{i}\right)^{2 m+1}}{\alpha_{1}^{2}+\sum_{i=2}^{n} \alpha_{i}^{2}\left(\lambda_{1} / \lambda_{i}\right)^{2 m}}\right) .
$$

Note that this expression converges to $\lambda_{1}^{-1}$ even in the case of a multiple eigenvalue.

To prevent $\boldsymbol{x}_{m}$ from approaching zero $\left(\lambda_{1}>1\right)$ or growing in magnitude $\left(\lambda_{1}<1\right), \boldsymbol{x}_{m}$ is normalized to a unit vector at each step. The choice of Euclidean norm $\|\cdot\|_{2}$ has two advantages over alternatives: it simplifies the computation of $r_{m}$, and it facilitates the deflation procedure described below.

The computational procedure represented by Eq. (5-1) may be used to find the $k$-th eigenvalue by simply projecting the initial estimate $\boldsymbol{x}_{0}$ onto the orthogonal complement of the first $k-1$ eigenvectors:

$$
\hat{\boldsymbol{x}}_{0}=\boldsymbol{x}_{0}-\sum_{i=1}^{k-1}\left(\boldsymbol{x}_{0}^{T} \boldsymbol{u}_{i}\right) \boldsymbol{u}_{i}
$$

so that $\hat{\boldsymbol{x}}_{0}^{T} \boldsymbol{u}_{j}=0$ for $j<k$. Then, for $\boldsymbol{x}_{0}=$ $\sum_{i=1}^{n} \alpha_{i} \boldsymbol{u}_{i}$, we have $\boldsymbol{x}_{0}^{T} \boldsymbol{u}_{i}=\alpha_{i}$ and

$$
\hat{\boldsymbol{x}}_{0}=\sum_{i=k}^{n} \alpha_{i} \boldsymbol{u}_{i}
$$

resulting in

$$
\begin{aligned}
\boldsymbol{x}_{m} & =\boldsymbol{T}^{-m} \hat{\boldsymbol{x}}_{0}=\sum_{i=k}^{n} \alpha_{i} \boldsymbol{T}^{-m} \boldsymbol{u}_{i}=\sum_{i=k}^{n} \alpha_{i} \lambda_{i}^{-m} \boldsymbol{u}_{i} \\
& =\lambda_{k}^{-m}\left(\alpha_{k} \boldsymbol{u}_{k}+\sum_{i=k+1}^{n} \alpha_{i}\left(\lambda_{k} / \lambda_{i}\right)^{m} \boldsymbol{u}_{i}\right) .
\end{aligned}
$$

Thus, provided $\alpha_{k} \neq 0, \boldsymbol{x}_{m} /\left\|\boldsymbol{x}_{m}\right\|_{2}$ approaches $\boldsymbol{u}_{k}$ (or, in the case of a multiple eigenvalue, it converges to an eigenvector in the same eigenspace). Further, an expression analogous to (5-2) shows that the Rayleigh quotients $r_{m}$ converge to $\lambda_{k}^{-1}$ as $m \rightarrow \infty$. In practice, due to numerical considerations, orthogonality must be maintained by applying the projections at each step or at least periodically. Refer to [Wilkinson 1965] for background on this method.

In order to represent the complex geometry of the regions, especially at the higher levels, our initial grid was necessarily quite fine. Thus, although the code allows for an arbitrary number of grid refinements (using bisection), we based our computations on just two grids. Initial estimates of the eigenvectors on the coarse grid were randomly generated, and initial estimates on the fine grid were obtained by piecewise bilinear interpolation from the coarsegrid solutions. The method of Successive Overrelaxation (SOR) [Young and Gregory 1973] was used to solve the linear systems on the coarse grid, and a sequence of multigrid V-cycles [Demmel 1997] with weighted Jacobi smoothing was used on the fine grid.

\section{CONCLUSION}

In this note we sought an organized way to get a qualitative and a quantitative understanding of essential spectra. Our test case was the negative Neumann laplacian on a Rooms and Passage region that is a member of a multi-purpose family of counterexamples in the theory of Sobolev embeddings, a subject of great importance in the study of partial differential equations. Since this laplacian does not have a compact inverse we were assured of the existence of at least one point of essential spectrum. We think we found at least one such point and we have indicated a sequence $\left\{\lambda_{k}\right\}_{k=1}^{\infty}$ any of which might be an eigenvalue or a point of essential spectra.

The first named author was introduced to phenomena surrounding Rooms and Passages regions by Desmond Evans, one of the authors of [Edmunds and Evans 1987]. From a private communication from Evans (concerning the case study of this note) we quote the following: '.. we still have no idea about the nature of the essential spectrum: does it consist of one point, or some interval or the whole positive real axis...'. We thank Evans for his frequent help but the present authors take full responsibility for any misleading inferences we may have made from our calculations. We do not preclude the possibility of obtaining essential spectra for this case study (helping to decide, perhaps, between Hypotheses 1 and 2) from [Hempel et al. 1991], but we reiterate that the main purpose of this note is the establishment of a framework for the computational determination of essential spectra. 


\section{REFERENCES}

[Adams 1975] R. A. Adams, Sobolev spaces, Pure and Applied Mathematics 65, Academic Press, New YorkLondon, 1975.

[Beurling 1989] A. Beurling, The collected works, vol. 2: Harmonic analysis, edited by L. Carleson et al., Birkhäuser, Boston, 1989.

[Demmel 1997] J. W. Demmel, Applied numerical linear algebra, Soc. Ind. App. Math., Philadelphia, PA, 1997.

[Edmunds and Evans 1987] D. E. Edmunds and W. D. Evans, Spectral theory and differential operators, Clarendon Press, Oxford, 1987.

[Hempel et al. 1991] R. Hempel, L. A. Seco, and B. Simon, "The essential spectrum of Neumann Laplacians on some bounded singular domains", $J$. Funct. Anal. 102:2 (1991), 448-483.
[Lapidus, Neuberger, Renka, and Griffith 1996] M. L. Lapidus, J. W. Neuberger, R. J. Renka, and C. A. Griffith, "Snowflake harmonics and computer graphics: numerical computation of spectra on fractal drums", Internat. J. Bifur. Chaos Appl. Sci. Engrg. 6:7 (1996), $1185-1210$.

[Neuberger 1997] J. W. Neuberger, Sobolev gradients and differential equations, Lecture Notes in Math. 1670, Springer, Berlin, 1997.

[Neuberger and Noid 1987] J. W. Neuberger and D. W. Noid, "Numerical calculation of eigenvalues for the Schrödinger equation, III", J. Comp. Chemistry 8 (1987), 459-461.

[Wilkinson 1965] J. H. Wilkinson, The algebraic eigenvalue problem, Clarendon Press, Oxford, 1965.

[Young and Gregory 1973] D. M. Young and R. T. Gregory, A survey of numerical mathematics, vol. II, Addison-Wesley, Reading, MA, 1973.

J. W. Neuberger, Department of Mathematics, University of North Texas, Denton, TX 76203 (jwn@unt.edu)

R. J. Renka, Department of Computer Science, University of North Texas, Denton, TX 76203 (renka@cs.unt.edu)

Received May 20, 1998; accepted in revised from October 23, 1998 